\title{
Performance Accuracy of Classification Algorithms for Web Learning System
}

\author{
L. Jayasimman, Ph.D. \\ Assistant Professor in Computer Science, \\ Srimad Andavan College of Arts and Science, \\ Tiruchirappalli
}

\begin{abstract}
This Research paper focused on Classification accuracy based on Users' Preferences from the Web Learning System. This comparative study considers various classification algorithms like j48, Random Tree, Random Forest, CART and Naive Bayes in the Web Learning System. It also focuses on Artificial Neural Network (ANN) algorithms. The classification accuracy is identified by user's requirements based on the cognitive input. In this research Neural Network approach like MLP, PMLP, GO PMLP and PSO PMLP algorithms are proposed and validated. These algorithms classify the user preferences of the Web Learning System. As the User Preferences have many potential applications, mining on the User Preferences of the Web Learning System users was contemplated. Based on the response of the current users, a decision tree induction algorithm is used to predict the requirements of future users.
\end{abstract}

\section{Keywords}

Online Learning, User Interface Design, User Preferences Artificial Neural Network, Artificial Neural Network, PMLP.

\section{INTRODUCTION}

Extraction of useful patterns and relationships from the existing data is certainly a huge need of the hour. Individuals, especially internet surfers wish to retrieve relevant information in a flexible manner so that understanding human behaviours at real-time is also becoming a vital part of data mining [1]. In this paper invoked the concepts such as Multilayer Perceptrons, Genetic Optimization (GO) and Particle Swarm Optimization (PSO) techniques are specifically analyzed. ANNs offer a capability to perform tasks outside the scope of traditional data processing methods. ANNs distinguish patterns within vast data sets and then generalize those patterns into recommended courses of action. The primordial distinction against the traditional algorithms is that the Neural Networks learn and are not programmed. ANN involves the selection of learning rules, transfer functions, summation functions, and methods to connect the neurons within the network. The contemporary pedagogy is positively tailoring itself with the technological advancements especially in providing access to information and knowledge worldwide [2]. ANN is successfully used to model the complex nature of hydrological system. ANN is relatively fast and flexible, and it is able to extract the relation between the inputs and outputs of a process without knowledge of the underlying physics. ANNs with sufficient hidden units are able to approximate any continuous function to any degree of accuracy by performing efficient training. The proliferation of Web-Based technologies has a significant impact on the education arena [3]. It is highly desirable to apprehend Web-Based Education (WBE) that encompasses all aspects and processes of education with the effective use of World Wide Web as a communication medium and supporting technology [4]. Extraction of useful patterns and relationships from the existing data is certainly a huge need of the hour. Individuals, especially internet surfers wish to retrieve relevant information in a flexible manner so that understanding human behaviours at real-time is also becoming a vital part of data mining [5]. In this section, the concepts such as Multilayer Perceptrons, Genetic Algorithm (GA) and Particle Swarm Optimization (PSO) techniques are concisely delineated.

\subsection{Parallel Multilayer Perceptron}

The categorization of the preferences of the web learning system could be performed in a more suitable manner with the biologically inspired classification algorithm, namely, neural network. The Multilayer Perceptrons are designed such that the input enters and passes through layer by layer, until it arrives at the optimal output [6]. The Parallel MLP accomplishes the classification of the preferences that exceeds the accuracy of MLP and other neural classifiers [7]. The implementation of parallelization in the data improves the classification accuracy and the reduction of time factor in training the same.

\subsection{Genetic Optimized PMLP}

On the account of surpassing the accuracy of the PMLP, the evolutionary avenue genetic optimization that aims at improving the solution by uncovering the best combination of input variables is sought [15]. The operators such as Selection, Mutation and Crossover were interactively fed forward to the input layers of PMLP. By the dint of genetic optimization of the learning rate and momentum, the process is intensified to the convergence of the output. As a result of the process of calculating fitness value, computing and selecting the offspring count, the cross-over and mutation, the optimized values are obtained.

To improve the performance of the PMLP, the parameters of the neural network is optimized using Genetic Optimization (GO). All the user preferences are classified using proposed neural network classifiers with an optimization technique. The cognitive attributes are used as the input for the new genetically optimized neural network.

\subsection{Particle Swarm Optimized PMLP}

PSO is another evolutionary computation technique developed by Eberhart and Kennedy in 1995, which was inspired by the social behavior of bird flocking and fish schooling. PSO is a swarm intelligence algorithm where particles fly through a constraint guided multidimensional search space, the best swarm particles, and their previous positions [9]. Velocity is the mechanism used for this movement [10]. This makes exploration of continuous space efficient and thorough with correct problem parameters. PSO has its roots in artificial life and social psychology, as well as in engineering and computer science. It utilizes a "population" of particles that fly through the problem hyperspace with given velocities. At each iteration, the velocities of the individual particles are sarcastically adjusted according to the historical best position 
for the particle itself and the neighborhood best position. Both the particle best and the neighborhood best are derived according to a user defined fitness function. The movement of each particle naturally evolves to an optimal or near-optimal solution.

Particle Swarm Optimization (PSO) approach has the ability to maximize the classification accuracy. PSO is an Artificial Intelligence (AI) technique based on the behavior of neural network system. PSO implementation improves the performance of MLP and it eliminates the drawbacks of MLP such as slow Convergence. Classification accuracy of an ANN and its ability are enhanced when PSO is applied in the design of a Neural Network. Particle Swarm Optimized Parallel Multi Layer Perceptron (PSO PMLP) is applied to classify the user preferences of Web learning system with cognitive aspect. New hybrid PSO-BP algorithm is an improvement over the Adaptive Particle swarm optimization algorithm (APSOA) and BP algorithm in convergent speed and Accuracy [8]. Particle Swarm optimization of the functions, namely, the learning rate and momentum improves the classification accuracy. When implementing the particle swarm algorithm, several considerations such limiting maximum velocity, selecting acceleration constants, the constriction factor, or the inertia constant must be taken into account to facilitate the convergence.

\section{RESEARCH METHODOLOGY}

In this Research, the users' preferences of Web Learning System parameters such as cognition and cognitive load are investigated principally. Investigations are carried out to study the efficiency of decision tree algorithms namely, Naïve Bayes, Random Forest, Random tree, J48 and CART. The corpus, consisting of data from both general cognition and cognitive load, is used as the input for the classification algorithms. The Random Forest and Random Tree give better results. This methodology based new frame work is capable of enhancing the classification accuracy of user preferences in a Web Learning System [10]. The below frame work based on various Neural Network classification techniques such as PMLP, GO PMLP and PSO PMLP provides enhanced classification accuracy of Web Learning System [18].

Further studies are conducted to investigate the classification accuracy based on Neural Network approach namely Multi Layer Perceptron (MLP). The classification accuracy of the neural algorithm proves to be better than the existing accuracy levels with decision tree classifiers. Hence, a framework comprising three classification algorithms is proposed to enhance classification accuracy [12]. The cognitive attributes are used as the training input for the decision tree induction algorithms, namely, Naïve Bayes, Random Forest, Random Tree, J48, CART and the Neural Network approach, MLP. It is observed from the results that, the Neural Network approach MLP gives enhanced classification accuracy when compared to other classifiers [19]. The new frame work is capable of enhancing the classification accuracy of user preferences in a Web Learning System. The frame work given in Figure 2.1 based on various Neural Network classification techniques such as PMLP, GO PMLP and PSO PMLP provides enhanced classification accuracy of Web Learning System.

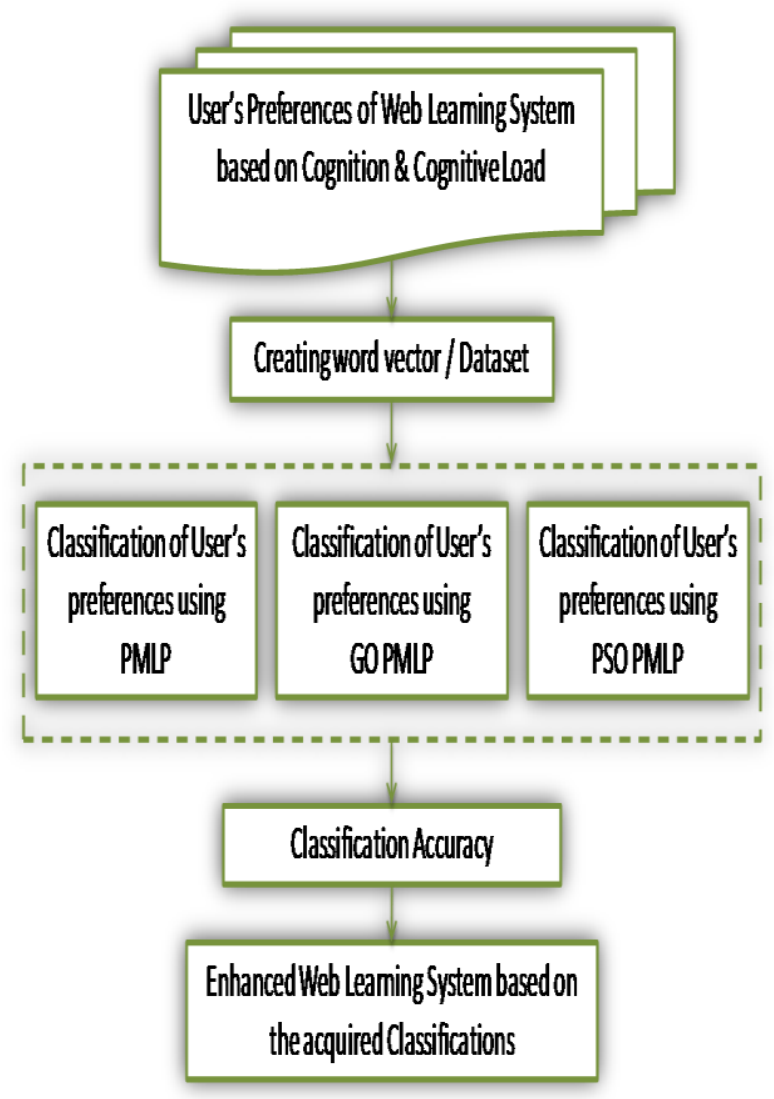

Figure: 2.1 Frameworks for Web Learning System

The classification accuracy of the neural algorithm proves to be better than the existing accuracy levels with decision tree classifiers. Hence, a framework comprising three classification algorithms is proposed to enhance classification accuracy [14].

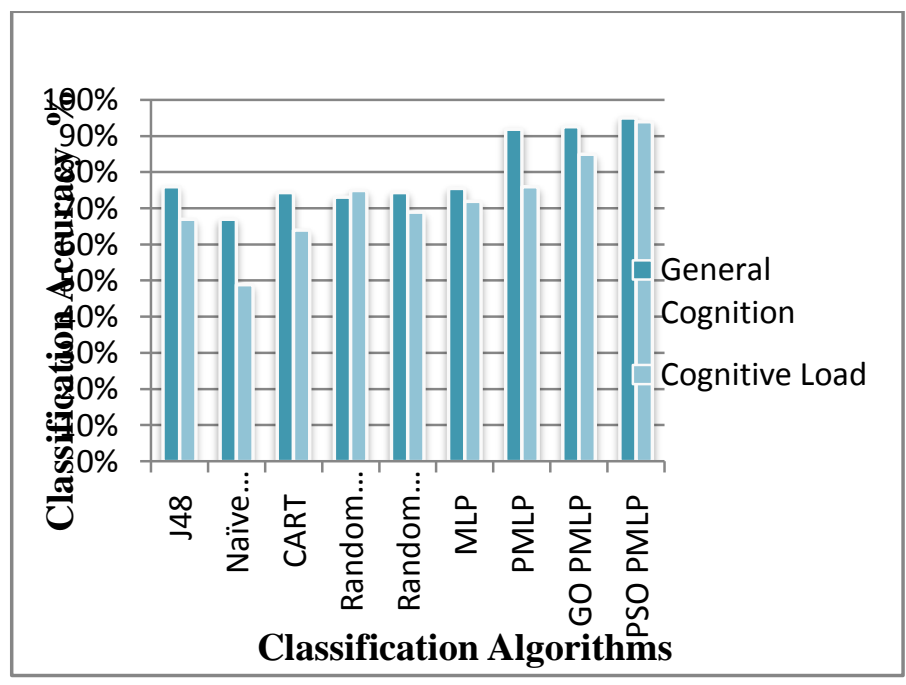

Figure 2.2 Classification Accuracy of Proposed Algorithms Vs. Existing Algorithms 


\subsection{Parallel Multi Layer Perceptron (PMLP)}

In this research, the cognitive attributes are used as the training input for a novel Parallel Multilayer Perceptron (PMLP) algorithm. In PMLP, the network is divided into blocks of adjacent neurons and each is allocated to a processor [16]. This approach to parallelization attempts to take advantage of the locality that exists between adjacent neurons. Parallelizing the hidden layer reduces the complexity of the connection which in turn also reduces the number of iterations. Gaussian and bipolar sigmoidal are the activation functions used in the proposed network.

\subsection{Parallel Multi Layer Perceptron with Genetic Optimization (GO PMLP)}

To enhance the classification accuracy of the proposed PMLP through genetic optimization is proposed. Genetic algorithm (GO) is based on biological evolution, which uses selection and reproduction operators to search for global optimum for a given problem. The intrinsic step involved in GO PMLP is that the learning rate and momentum are optimized with genetic optimization.

\subsection{Parallel Multi Layer Perceptron with Particle Swarm Optimization (PSO PMLP)}

The Particle Swam Optimization MLP (PSO PMLP) algorithm is proposed to improve the classification accuracy. PSO is a swarm based optimization method based on flocking behaviour of birds, evolving an optimal solution for a problem [17]. The parameters for PSO PMLP and GO PMLP are same. The essential step involved in PSO PMLP is the optimization of learning rate and momentum. The significant improvement in the classification accuracy, while implementing GO PMLP and PSO PMLP, is identified for the Cognitive behaviour and load. Table 3.1 and Figure 3.2 show that the proposed three Neural Network based algorithms enhance the classification accuracy when compared to existing classification algorithms.

\section{RESULTS AND FINDINGS}

The significant improvement in the classification accuracy, while implementing GO PMLP \& PSO PMLP, is identified for the cognitive behaviour and cognitive load. Table 3.1 shows the efficiency of decision tree algorithms namely, Naïve Bayes, Random Forest, Random tree, J48 and CART, the neural network algorithm MLP and the proposed algorithms such as PMLP,GO PMLP,PSO PMLP. The corpus, consisting of data from both general cognition and cognitive load, is used as the input for the classification algorithms. The Random Forest and Random Tree give better results. Figure 3.1 show that the proposed three Neural Network based algorithms enhance the classification accuracy when compared to existing classification algorithms.

Table 3.1. Classification Accuracy of Proposed Vs. Existing Algorithms

\begin{tabular}{|l|l|l|}
\hline $\begin{array}{l}\text { Data Mining } \\
\text { Algorithm }\end{array}$ & $\begin{array}{l}\text { General } \\
\text { Cognition }\end{array}$ & $\begin{array}{l}\text { Cognitive } \\
\text { Load }\end{array}$ \\
\hline J48 & $76 \%$ & $67 \%$ \\
\hline Naïve Bayes & $67.07 \%$ & $49 \%$ \\
\hline CART & $74.39 \%$ & $64 \%$ \\
\hline
\end{tabular}

\begin{tabular}{|l|l|l|}
\hline Random Forest & $73.17 \%$ & $75 \%$ \\
\hline Random Tree & $74.39 \%$ & $69 \%$ \\
\hline MLP & $75.6 \%$ & $72 \%$ \\
\hline PMLP & $92 \%$ & $76 \%$ \\
\hline GO PMLP & $92.68 \%$ & $85 \%$ \\
\hline PSO PMLP & $95.12 \%$ & $94 \%$ \\
\hline
\end{tabular}

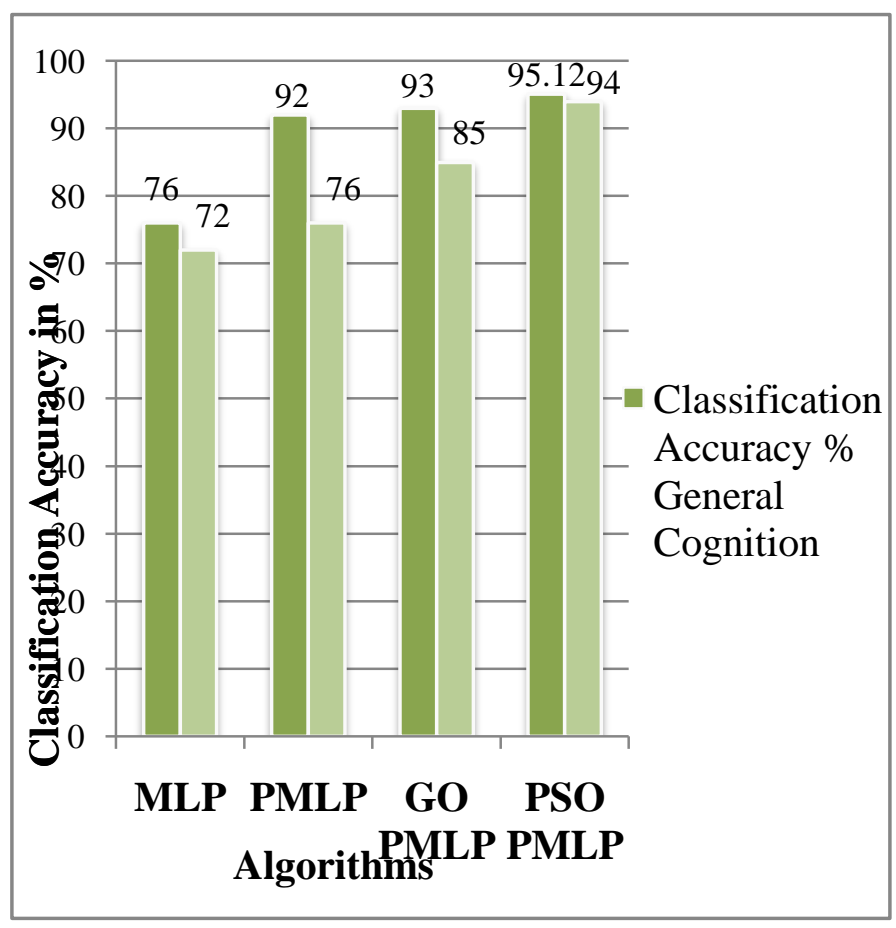

Figure 3.2 Comparison of PSO PMLP

Based on the above analysis it is obvious to note down that the proposed PSO PMLP classifier surpasses the existing MLP, PMLP, GO PMLP classifiers. The classification accuracy obtained by PSO PMLP measures to $95.12 \%$ for General Cognition and 94\% for Cognitive Load. The results are shown graphically in Figure 3.1

\section{CONCLUSION}

The cognitive attributes are used as the training input for the decision tree induction algorithms, namely, Naive Bayes, Random Forest, Random Tree, J48, CART and the Neural Network approach, MLP. The classification accuracy in identifying the user's requirements based on the cognitive input is recognized. It is observed from the results that, the Neural Network approach MLP gives enhanced classification accuracy when compared to other classifiers. The PMLP, GO PMLP and PSO PMLP algorithms are proposed and validated. These algorithms classify the user preferences of Web Learning System with enhanced classification accuracy and the overall framework also helps the same. Experimental results reveal that the classification accuracy of the Neural Network improves significantly. The PMLP gives the classification accuracy of $92 \%$ for general cognition and $76 \%$ for cognitive load. The Genetic Optimized PMLP achieves classification accuracy of $92.68 \%$ for cognition and $85 \%$ for 
cognitive load, for Web Learning data set. The PSO PMLP achieves better results than Genetic Optimized PMLP with the proposed new classification system provides individual recommendations in accordance with the analysis of single Users' searching custom and interest, so the quality of educational service could be improved.

\section{REFERENCES}

[1] Marian Simko, Michal Barla, Maria Bielikova, "ALEF: A Framework for Adaptive Web-based Learning 2.0", International Scientific Conferences on The World Computer Congress, pp.1-12, 2010.

[2] Joseph A. Konstan Ricardo Conejo José L. Marzo Nuria Oliver (Eds.) "User Modeling, Adaption, and Personalization 19th International Conference", Springer , UMAP 2011 Girona, Spain, July 11-15, 2011

[3] Shaharuddin Md Salleh, Zaidatun Tasir, Nurbiha A Shukor "Web-Based Simulation Learning Framework to Enhance Students' Critical Thinking Skills", International Educational Technology Conference on Procedia - Social and Behavioral Sciences, Volume 64, pp. 372-381, 2012.

[4] Mustafa, K. O. C. “ Individual learner differences in web-based learning environments: From cognitive, affective and social cultural perspectives", Turkish Online Journal of Distance Education-TOJDE, Volume 6, Issue 4, pp. 1257-1261, 2005.

[5] Abdullah H. Wahbeh, Qasem A. Al-Radaideh, Mohammed N.Al-Kabi, Emad M. Al-Shawakfa, "A Comparison Study betweenmData Mining Tools over some Classification Methods" International Journal of Advanced Computer Science and Applications, Special Issue on Artificial Intelligence, pp. 18-26, 2011.

[6] Hong jun Lu, Rudy Setiono, Huan Liu, "Effective Data Mining Using Neural Networks", IEEE transactions on knowledge and data engineering, Volume 8, Number 6 , pp. 957-961, 1996.

[7] G. Saravana kumar, P.K.Kalra, "Optimization by Neural Networks", Quarterly Scientific Magazine of IIT Kanpur, Volume 6, Number 3, pp.87-91, 2004.

[8] D. Montana, "Introduction to the Special Issue: Evolutionary Algorithms for Scheduling", Evolutionary Computation, Volume 6, Number 1, 1998.

[9] Cristobal Romero, Sebastian Ventura, Carlos De Castro, Wendy Hall, Muan Hong Ng, Hong Ng "Using Genetic Algorithms for Data Mining in Web based Educational Hypermedia Systems", Proceedings of AH2002 workshop Adaptive Systems for Web based education, pp. 137-142, 2002.
[10] Qinghai Bai, "Analysis of Particle Swarm Optimization Algorithm", Computer Science and Informatics, Volume 3, Issue 1, pp 180-184, 2010.

[11] Maria-Iuliana DASCALU, "Application of Particle Swarm Optimization to Formative E-Assessment in Project Management", Informatica Economica, volume 15, Number 1, pp 48-59, 2011.

[12] Mona Alkhattabi, Daniel Neagu, Andrea Cullen, "Information Quality Framework for e-Learning Systems", Knowledge Management \& E-Learning: An International Journal, Volume 2, Number 4, pp. 340-362, 2013.

[13] Biplab Kanti Das, Saurabh Pal, "A framework of Intelligent Tutorial System to incorporate adaptive learning and assess the relative performance of adaptive learning system over general classroom learning", International Journal of Multimedia and Ubiquitous Engineering, Volume 6, Number 1, pp. 43-54, 2011.

[14] Gwo-Jen Hwang, Peng-Yeng Yin, Tzu-Ting Wang, Judy C.R.Tseng, Gwo-Haur Hwang "An enhanced genetic approach to optimizing auto-reply accuracy of an elearning system" Computers and Education, Volume 51, pp. 337-353, 2008.

[15] Kuok King Kuok, Sobri Harun, Siti Mariyam Shamsuddin, Po-Chan Chiu, "Evaluation of Daily Rainfall-Runoff Model Using Multilayer Perceptron and Particle Swarm Optimization Feed forward Neural Networks", Journal of Environmental Hydrology, Volume 18, pp. 1-16, 2010.

[16] Neeraj Bhargava, Atif Aziz, Rajiv Arya "Selection Criteria for Data Mining Software: A Study", International Journal of Computer Science Issues, Vol. 10, Issue 3, No 2, May 2013, ISSN (Print): 1694-0814 ISSN (Online): 1694-0784

[17] L.Jayasimman, "A Framework to Enhance Classification Accuracy for Web Learning System", International Journal of Computer Science and Telecommunications, UK, Volume 5, Issue 11, November 2014, ISSN $2047-$ 3338 .

[18] L.Jayasimman, E.George Dharma Prakash Raj, "A Soft Computing Approach for User Preference in Web Based Learning", International Journal of Computer Applications,. Volume 61- No.21, January 2013.

\section{AUTHOR'S PROFILE}

L. Jayasimman working as a Assistant Professor, Department of Computer Science, Srimad Andavan Arts \& Science College, Trichy, India. He received his M.Tech degree in Bharathidasan University, Trichy, India in 2008 and completed his $\mathrm{PhD}$ (Computer Science) in Bharathidasan University in 2014. 